\title{
Embolisation of Orbital Varix via the Superficial Temporal Vein
}

\author{
N. MAVILIO, A. PAU*, R. PISANI* , A. CASASCO**, M. ROSA \\ Department of Neuroradiology, San Martino Hospital; Genova, Italy \\ * Department of Neurosurgery; University of Genova, Italy \\ ** Department of Diagnostic and Interventional Neuroradiology, Groupe Hospitalier Pitie-Salpetriere; Paris, France
}

Key words: orbital varix, superficial temporal vein, superior ophthalmic vein, orbital phlebography, embolisation, Guglielmi detachable coils

\section{Summary}

A case of orbital varix is presented, in which the lesion was successfully treated by means of embolisation with Guglielmi detachable coils, via the superficial temporal vein. Reports of endovascular treatment via transvenous route of orbital varix are lacking, only one case having previously been presented in the literature. We stress endovascular management for such an entity.

\section{Introduction}

Orbital varices are relatively uncommon congenital venous anomalies, their main symptoms being ipsilateral intermittent exophthalmos, orbital pain, diplopia, and sometimes reduced visual acuity. Only one case of transvenous embolisation of an orbital varix has previously been described in the literature ${ }^{1}$. In this patient the orbital varix was approached and embolised, on two occasions, through the internal jugular vein, the inferior petrosal sinus, the cavernous sinus and the superior ophthalmic vein. We report the first documented case in which an orbital varix was embolised with Guglielmi detachable coils (GDCs) via the external jugular vein, the superficial temporal vein, and the angular vein.

\section{Case report}

This 35-year-old man presented with a 14year history of right intermittent exophthalmos and diplopia. At ophthalmologic examination, visual acuity, ocular movement, field and fundus were unremarkable. No bruit or pulsation was present. Valsalva maneuver evoked 4-mm right eye proptosis and double vision in vertical elevation. Orbital CT scan revealed a right retrobulbar intraconic mass with mild contrast enhancement (figure 1).

Right internal and external angiography was normal. Right orbital phlebography through percutaneous puncture of the frontal vein indicated a voluminous orbital varix in correspondence of the third segment of the superior ophthalmic vein (figure 2).

A supraorbital anastomotic channel between the superficial temporal vein and the angular vein was also evident. Attempts at catheterization of the inferior petrosal sinus failed due to the anatomical variance of the sinus itself, which was replaced by a fine venous network. The superior ophthalmic vein was then approached via percutaneous puncture of the external jugular vein, the superficial temporal vein and the angular vein (figure 3 ).

The instrumentation used to reach the varix included the 4F Terumo introducer, the Rapid 


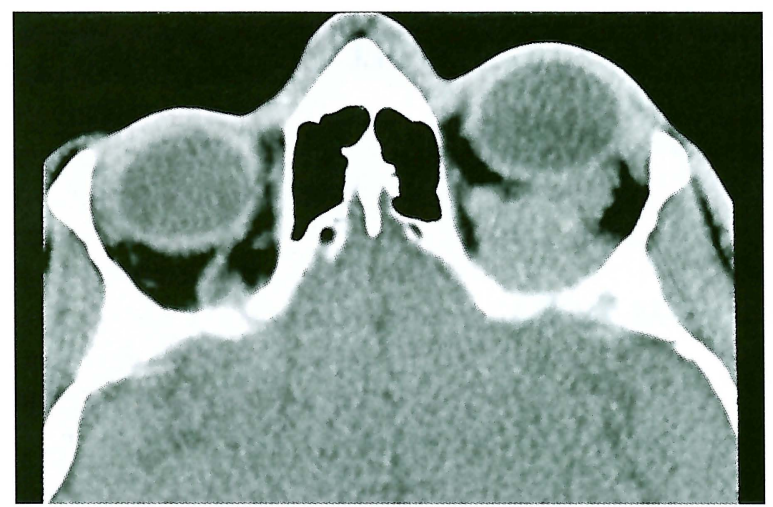

Figure 1 Contrast enhanced orbital CT scan, performed during Valsalva maneuvre, shows the right proptosis and retrobulbar intraconic mass.

Transit 2 Markers microcatheter and the 0.016Terumo GT guidewire. Subtotal embolisation of the varix was achieved by means of two $7 \mathrm{x}$ $30 \mathrm{~cm}$ and one $8 \times 20 \mathrm{~cm}$ GDCs (figure 4).

Concurrent phlebographic controls during the procedure were maintained through percutaneous injection of the frontal vein (figure 5).

The embolisation was deliberately subtotal because of the likely risks of completely occluding the ophthalmic superior vein and because of an increase in proptosis at the end of the procedure. Proptosis completely disappeared about three hours later. The following

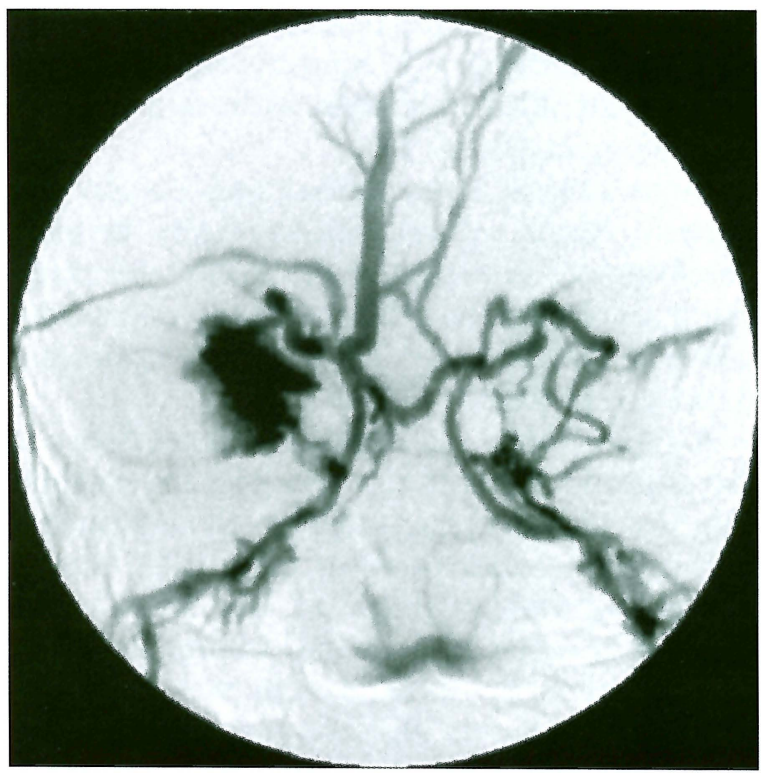

Figure 2 Right anteroposterior orbital phlebography via the frontal vein reveals the large orbital varix of the third segment of the superior ophthalmic vein. postoperative course was uneventful. At discharge, 5 days later, no spontaneous or induced exophthalmos was present. After one year, the patient is well, his ophthalmologic examination is negative, except for a slight and occasional diplopia in extreme direction of vision towards the top. Phlebographic control via the frontal vein indicates a further reduction in size of the small residue of the varix and a narrowing of the ophthalmic superior vein (figure 6).

\section{Discussion}

No large series of patients with orbital varices has ever been published. Therefore guidelines to management of orbital varices are still controversial. Conservative treatment may be mainly advocated in asymptomatic or poorly symptomatic cases. Indications for active management include severe pain, severe proptosis, compressive optic neuropathy due to orbital mass effect, recurrent bleeding and thrombosis.

Moreover, varix treatment may be undertaken to remedy cosmetic disfigurement. Surgical excision through lateral orbitotomy or frontotemporal craniotomy has been performed with ligature, bipolar or laser cautery, and haemostatic clips ${ }^{2,3}$, even if in most cases it has not been possible for the surgeon to remove the varix

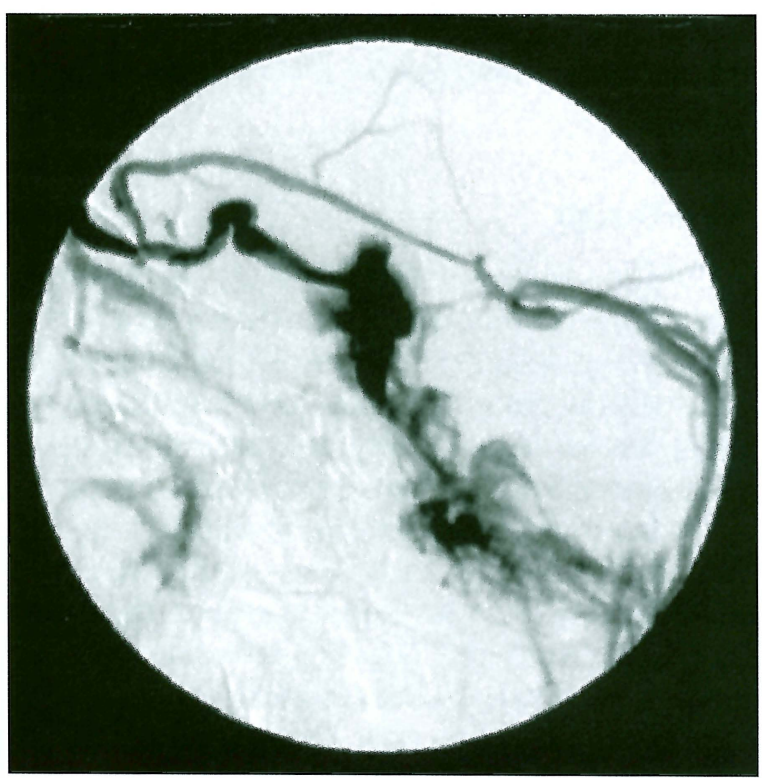

Figure 3 Selective injection of the temporal superficial vein shows the supraorbital anastomosis with the angular vein, the superior ophthalmic vein, and the orbital varix (lateral projection). 

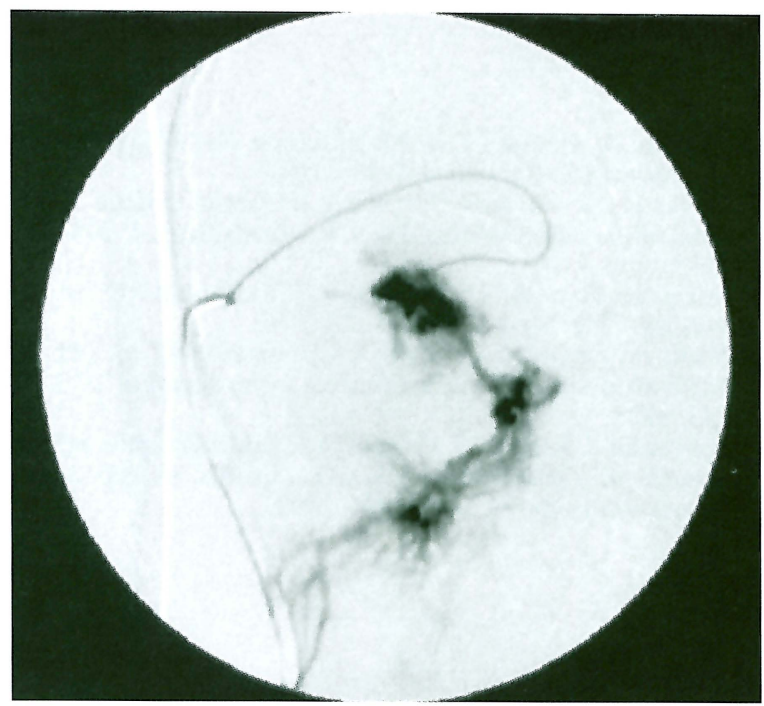

Figure 4 Selective catheterization and injection of the orbital varix through the superficial temporal vein (lateral view).

completely. In fact, open surgery tends to be punctuated by intraoperative complications such as profuse bleeding, and difficult dissection of the thin varix wall. Bleeding may obscure the fine branches of the motor ocular nerves, which may be inadvertently sectioned.

Furthermore, orbital fat scarring may lead to iatrogenic enophthalmos. Hence, surgical management has to be contraindicated in patients with haemorrhagic tendency, in poor health, younger then ten years of age or in geriatric patients $^{3}$. Recently, in order to reduce intraoperative bleeding and to facilitate surgical excision, direct puncture and embolisation of the varix, after surgical exposure by craniotomy, was proposed ${ }^{4}$. In the case report of Takechi ${ }^{1}$, the transvenous route to the inferior petrosal sinus, routinely used for hypophyseal hormonal samples or for embolisation of dural arteriovenous fistulae of the cavernous sinus, was successfully employed. Unfortunately, as stressed by these authors, this approach may be technically difficult and in some cases unfeasible because of developmental variations of the inferior petrosal sinus.

In our case, after failed attempts to catheterize the inferior petrosal sinus, we decided to resort to supraorbital anastomosis between the superficial temporal vein and the angular vein. Catheterization of the superior ophthalmic vein and of the varix was thus possible. We preferred to perform subtotal embolisation of the

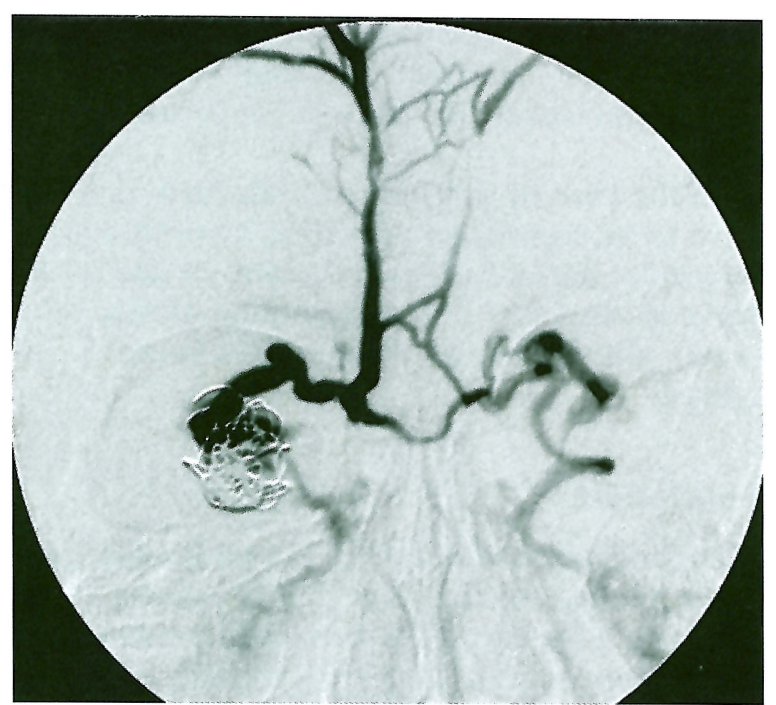

Figure 5 Anteroposterior intraoperative phlebographic control, via the frontal vein, indicates the subtotal embolisation of the orbital varix.
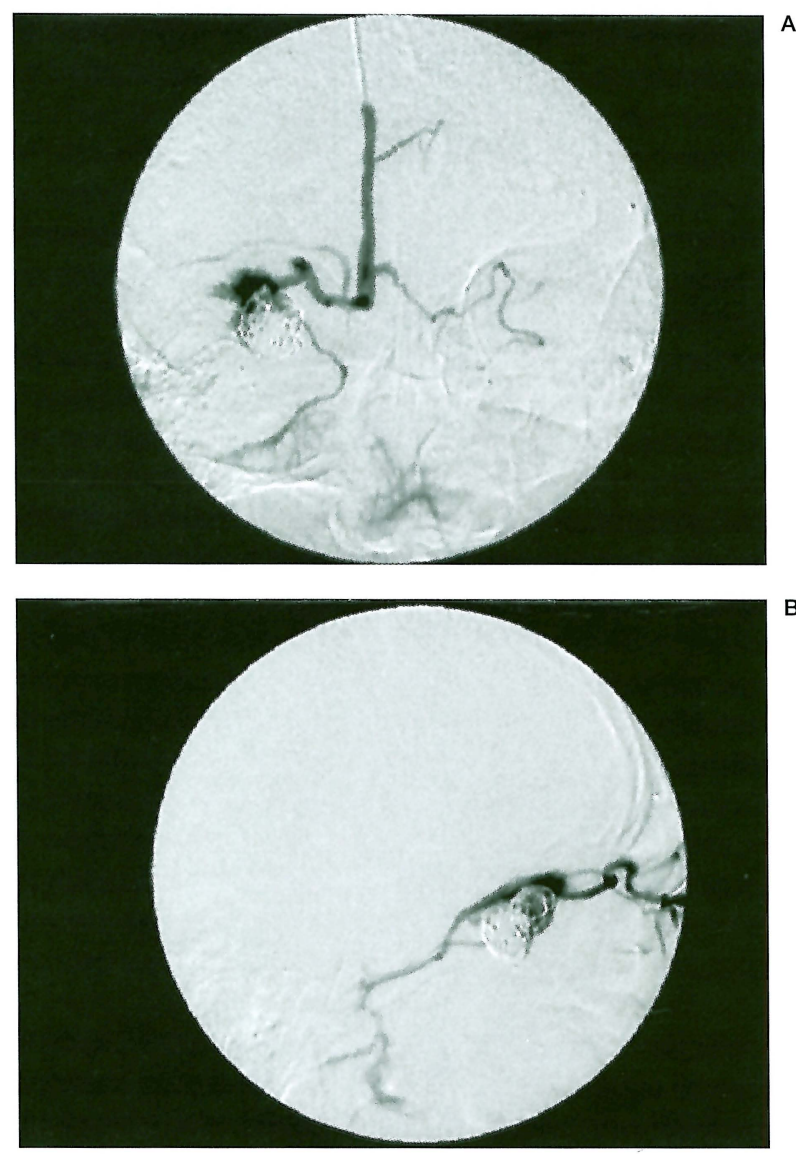

Figure 6 Anteroposterior (A) and lateral (B) phlebographic control, via the frontal vein, performed one year after the embolisation. A further reduction of the small residue of the orbital varix is evident. 
varix since, as reported elsewhere ${ }^{5}$, complete embolisation of such vascular lesions may sometimes result in damage to visual structures.

In the case of subtotal embolisation, patients have to be controlled over time by orbital phlebography, and if further reduction in size of the varix is observed or symptomatology does not recur (our case), no further treatment is required.

\section{Conclusions}

Embolisation with GDCs is a minimally traumatic procedure that has clearly proved to be safe and effective. Our own and previous reports demonstrate that this method of treatment may be successfully adopted in orbital varices, via the transvenous route, even if observations on larger case series are required to collect more information on this issue. Attempts at endovascular management are strongly recommended prior to surgical excision.

\section{References}

1 Takechi A, Uozumi T et Al: Embolisation of orbital varix. Neuroradiology 36: 487-489, 1994.

2 Beyer R, Levine MR, Sternberg I: Orbital varices: a surgical approach. Ophthal Plast Reconstr Surg 1: 205-210, 1985.

3 Song G, Tian W et Al: Surgical treatment of orbital varices. Chin Med J 92: 723-728, 1978.

4 Weill A, Cognard C et Al: Embolization of an orbital varix after surgical exposure. Am J Neuroradiol 19: 921923, 1998.

5 Rootman J, Kao SCS, Graeb DA: Multidisciplinary approach to complicated vascular lesions of the orbit. Ophthalmology 99: 1440-1446, 1992.

Dr Nicola Mavilio Servizio di Neuroradiologia Ospedale San Martino Largo Rosanna Benzi, 10 I-16132 Genova (Italy) 